\title{
Genetic Control and Phenotypic Characterization of Panicle Architecture and Grain Yield Related Traits in Foxtail Millet (Setaria Italica)
}

Hui Zhi

Institute of Crop Sciences, Chinese Academy of Agricultral Sciences

\section{Qiang He}

Chinese Academy of Agricultural Sciences Institute of Crop Sciences

Sha Tang

Chinese Academy of Agricultural Sciences Institute of Crop Sciences

Junjun Yang

Chinese Academy of Agricultural Sciences Institute of Crop Sciences

\section{Wei Zhang}

Chinese Academy of Agricultural Sciences Institute of Crop Sciences

Huifang Liu

Chinese Academy of Agricultural Sciences Institute of Crop Sciences

Yanchao Jia

Chinese Academy of Agricultural Sciences Institute of Crop Sciences

Guanqing Jia

Chinese Academy of Agricultural Sciences Institute of Crop Sciences

Aiying Zhang

Institure of millet crops, Shanxi Agricultural University

Yuhui Li

Institute of millet crops, Shanxi Agricultural University

Erhu Guo

Institute of millet crops, Shanxi Agricultural University

Ming Gao

Institute of Crop Sciences, Jilin Academy of Agricultural Sciences

Shujie Li

Institute of Crop Sciences, Jilin Academy of Agricultural Sciences Gongzhuling Branch

Junxia Li

Cereal Crops Institute, Henan Academy of Agricultural Sciences

$\mathrm{Na}$ Qin

Cereal Crops Institute, Henan Academy of Agricultural Sciences

\section{Cancan Zhang}

Cereal Crops Institute, Henan Academy of Agricultural Sciences

\section{Chunye Ma}

Cereal Crops Institute, Henan Academy of Agricultural Sciences

Haijin Zhang

Institute of Dry-land Agriculture and Forestry, Liaoning Academy of Agricultural Sciences

\section{Guoqiu Chen}

Institute of Dry-land Agriculture and Forestry, Liaoning Academy of Agricultural Sciences

\section{Wenfei Zhang}

Institute of Dry-land Agriculture and Forestry, Liaoning Academy of Agricultural Sciences

\section{Haigang Wang}

Center for Agricultural Genetic Resources Research, Shanxi Agricultural University

Zhijun Qiao

Center for Agricultural Genetic Resources Research, Shanxi Agricultural Universty 


\section{Lu Xing}

Aanyang Academy of Agricultural Sciences

\section{Sunying Wang}

Anyang Academy of Agricultural Sciences

Jinrong Liu

Anyang Academy of Agricultural Sciences

\section{Jun Liu}

Chinese Academy of Agricultural Sciences Institute of Crop Sciences

Xianmin Diao ( $\square$ diaoxianmin@caas.cn )

Institute of Crop Sciences, Chinese Academy of Agricultural Sciences https://orcid.org/0000-0002-8957-4101

\section{Research Article}

Keywords: Setaria italica, Panicle architecture, Grain yield, QTL

Posted Date: February 16th, 2021

DOI: https://doi.org/10.21203/rs.3.rs-159458/v1

License: (c) (i) This work is licensed under a Creative Commons Attribution 4.0 International License. Read Full License 


\section{Abstract}

Panicle architecture and grain weight, both of which are influenced by genetic and environmental factors, have significant effects on grain yield potential. Here, we used a recombinant inbred line population (RIL) of 333 lines, which were grown in 13 trials with varying environmental conditions, to identify quantitative trait loci (QTL) that control differences in 9 agronomic traits related to panicle architecture and grain yield. We find that panicle weight, grain weight per panicle, panicle length, panicle diameter, and panicle exsertion length varied across different geographical locations. QTL mapping revealed 159 QTL for nine traits, of these, 34 QTL were identified in 2 to 12 environments, suggesting that the genetic control of panicle architecture in foxtail millet is sensitive to photoperiod or other environmental factors. 88 QTL controlling different traits formed 34 co-located QTL clusters, including the triple QTL cluster qPD9.2/qPL9.5/qPEL9.3, the genomic region of which was detected by 23 times in 13 environments. Several candidate genes were identified in the genomic intervals of multi-environmental QTL or colocated QTL clusters, including Seita.2G388700, Seita.3G136000, Seita.4G185300, Seita.5G241500, Seita.5G243100, Seita.9G281300, and Seita.9G342700. Among these, Seita.9G342700 was the candidate gene of qPD9.2/qPL9.5/qPEL9.3 QTL cluster, it is homologous to rice OSMADS56, which encodes a putative MADS-box transcription factor that determines inflorescence architecture in rice. These results not only provided a basis for further fine mapping, functional studies and marker-assisted selection of panicle architecture related traits in foxtail millet, but also benefited comparative genomics of cereal crops.

\section{Introduction}

Foxtail millet (Setaria italica) is one of the most important cereal crops that domesticated in China about 10,000 years ago (Hu et al. 2018; Lu et al. 2009). The mature panicle of foxtail millet contains many primary branches that are attached to the main axis (often referred to as the rachis), several secondary branches on the primary branches, tertiary branches on the secondary branches, and each of the tertiary branches bears numbers of spikelets (grains). To improve grain yield per unit growing area by cultivating varieties with large panicles, which have long branches, more grain number, and enlarged grains, is a major goal of foxtail millet breeding. Despite a significant progress has been made in foxtail millet grain production during a 40-year scientific breeding in China (Diao et al. 2014), the molecular and genetic mechanisms underlying the grain yield, especially panicle architecture, in foxtail millet remain unclear.

Most of foxtail millet cultivars have only one tiller, which bears one panicle (Doust 2007). Panicle architecture, such as panicle length (PL), panicle diameter (PD), primary branch number (PBN), primary branch length (PBL), and grain number per panicle (GNP) mainly determine grain yield per plant. Panicle architecture is mainly determined by the fate of a meristem, and the timing of the meristem phase shift from the branch meristem to the spikelet meristem (Kyozuka et al. 2014). Many genes involved in CLAVATA-WUS signaling pathway, such as FLORAL ORGAN NUMBER 1 (FON1)(Suzaki et al. 2004), FON4 (Chu et al. 2006), OsWUS, ZmWUS, ZmWUS2 (Nardmann and Werr 2007), thick tassel dwarf 1 (TD1) (Bommert et al. 2005), and many MADS-box transcription factors, including OsMADS34 (Gao et al. 2010), OsMADS14, OSMADS15, and OSMADS18 (Kobayashi et al. 2012), regulate panicle architecture by regulating meristem size and specification of meristem identity. Additionally, the MADS-box genes can also regulate inflorescence branching by repressing the expression of REDUCED CULM NUMBER 4 (RCN4), a rice TERMINAL FLOWER 1 (TFL 1)/CENTRORADIALIS homolog (Liu et al. 2013). Overexpression of TFL 1 homologs (RCN1 and RCN2) in rice (Nakagawa et al. 2002) and CENTRORADIALIS 1 (ZCN1) to ZCN6 in maize (Danilevskaya et al. 2010) delay the changes from branch shoot to floral meristem and lead to a highly branched inflorescence. Gain of function mutations in DENSE AND ERECT PANICLE1 (DEP1) enhance meristematic activity, resulting in a reduced length of the inflorescence internode, and increased number of grains per panicle (Huang et al. 2009a). In addition, mutations in DEP2 and DEP3 also exhibit the characteristic erect panicle phenotype and increased panicle length and grain size (Qiao et al. 2011; Zhu et al. 2010). Phytohormones, including auxin, cytokinin, and gibberellin, play essential roles in regulating inflorescence meristem identity, initiation, and enlargement. Loss of function mutations in genes that participate in local auxin biosynthesis, signaling and transport significantly affect panicle architecture development (Andrea et al. 2004; Komatsu et al. 2003; Komatsu et al. 2001; Phillips et al. 2011). Grain number 1a (Gn1a) encodes a cytokinin oxidase/dehydrogenase (OsCKX2) that degrades cytokinin, loss of function mutations in Gn1a accumulate higher levels of cytokinin in inflorescence meristems, resulting in a larger number of branches and spikelets (Ashikari et al. 2005). By contrast, the dysfunction mutation of LONELY GUY (LOG), which encodes an enzyme catalyzing the conversion of inactive cytokinin nucleotides to the active free-base forms, displays a small inflorescence with a decreased number of branches and spikelets (Kurakawa et al. 2007). Class 1 KNOTTED 1-like homeobox (KNOX) genes, such as Oryza sativa homeobox 1 (OSH1) (Tsuda et al. 2011), maize knotted 1 (KN1)(Vollbrecht et al. 2000), and Arabidopsis SHOOT MERISTEMLESS (STM) (Long et al. 1996), play a central role in promoting shoot apical meristem identity by decreasing the levels of GA and increasing the amount of cytokinin. Due to a KNOX-mediated transcriptional feedback loop, overexpression of Grain Number per Panicle1 (GNP1), which encodes rice GA20ox1, increases grain number and yield by increasing cytokinin activity in rice panicle meristems (Wu et al. 2016).

However, compared to the major crops, such as maize and rice, only a few genes associated with panicle architecture had been cloned and molecularly characterized in detail from Seteria mutants. By a screen for visible inflorescence mutant phenotypes via an N-nitroso-Nmethylurea (NMU) mutagenesis of S. viridis, Huang et al. (2017) found two sparse panicle mutants, spp1 and spp3. Both of them had 
disruptive mutations in SVAUX1 (AUXIN1) gene. Further study revealed that loss of function mutations in SVAUX1 and ZmAUX1 disrupt the inflorescence branch development in Setaria viridis and maize, and lead to sparse panicle phenotypes. In previous studies, we isoloated two mutants with abnormal panicle architecture from the EMS mutant library constructed by our lab. One is the loose-panicle mutant, a single G-toA transition in the fifth intron of the WRKY transcription factor results in three disorganized splicing events in mutant plants, and leads to lax primary branching pattern and aberrant branch morphology (Xiang et al. 2017). The other is simads34, an alternative splicing event caused an early termination codon in SiMADS34 results in an increased panicle width, primary branch length, and number of primary branches, but decreased panicle length and grain weight in simads34 compared with wild-type plants (Hussin et al. 2020).

From forward genetic studies, many QTL associated with panicle architecture or grain yield have been identified from various bi-parental populations. Doust et al. (2005) undertook QTL analysis of inflorescence differences between the foxtail millet and green foxtail; they detected 14 replicated QTL for primary branch number and density, spikelet number, and bristle number. By using a $F_{2}$ population derived from different foxtail millet cultivars, Fang et al. (2016) identified 12 QTL for main panicle length (PL), main panicle diameter (PD), panicle weight per panicle (PW), grain weight per panicle (GWP), and 1000-grain weight (TGW). Wang et al. (2017) identified 11 major QTL for eight agronomic traits, including 5 QTL for PL, PD, and PW. Wang et al. (2019) mapped 57 QTL for 11 agronomic traits, including 31 QTL for PD, PEL, PW, grain weight, and TGW. From different RIL populations, Liu et al. (2020) detected 47 QTL for straw weight, PW, GWP and TGW; Zhang et al. (2017) detected 32 QTL for PW, PL, PD, CN, TGW, and panicle exsertion length (PEL). Using a natural population. Jia et al. (2013) phenotyped 916 varieties under five different environments and identified 512 loci associated with 47 agronomic traits, including 39 loci for PW, PL, PD, primary branch number (PBN), and TGW. Jaiswal et al. (2019) performed genome wide assocaition studies (GWAS) on 10 agronomic traits using 142 foxtail millet accessions, and identified 17 and 10 loci for grain yield and TGW, respectively. However, neither of the above QTL has been cloned, nor of the candidate genes located in the QTL intervals have been isolated, that hinder the understanding the mechanisms of panicle architecture and grain size development.

In the present study, a RIL population was developed from derivatives of hybrid between foxtail millet cultivar Ai 88 and Liaogu 1, among which their panicle architecture varied greatly. A large-scale and multi-environment analysis by using the RIL population was carried out, with an ultra-high density genetic map, to investigate the genetic control of panicle architecture and yield related agronomic traits in 13 environments. QTL mapping identified 159 QTL, in which intervals contain many candidate genes involved in panicle development. These favorable QTL alleles from either parent will be of great value to optimize panicle architecture breeding and increase grain yield.

\section{Materials And Methods}

\section{Plant materials}

A foxtail millet RIL population comprised of 333 lines was used in this study. This RIL population was generated from a cross between a backbone line Ai88 and an elite variety Liaogu1 as described by He et al. (2020). From 2015 to 2018, the RIL population was grown in seven geographical locations (Figure 1 and Table S1). Nanbin Farm (NB, coordinates: $109.19^{\circ} \mathrm{E} / 18.37^{\circ} \mathrm{N}$, Hainan Province) represents a short-day photoperiod location where the daily sunshine was $<12$ hours within the plant growing season. Six Northern-China locations, including Zhengzhou (ZZ, coordinates: $113.64^{\circ} \mathrm{E} / 34.75^{\circ} \mathrm{N}$, Henan Province), Changzhi (CZ, coordinates: $113.13^{\circ} \mathrm{E} / 36.20^{\circ} \mathrm{N}$, Shanxi province), Taiyuan (TY, coordinates: $112.55^{\circ} \mathrm{E} / 37.88^{\circ} \mathrm{N}$, Shanxi Province), Shunyi (SY, coordinates: $116.66^{\circ} \mathrm{E} / 40.13^{\circ} \mathrm{N}$, Beijing), Chaoyang (CY, coordinates: $120.46^{\circ} \mathrm{E} / 41.58^{\circ} \mathrm{N}$, Liaoning Province), and Gongzhuling (GZL, coordinates: $124.83^{\circ} \mathrm{E} / 43.51^{\circ} \mathrm{N}$, Jilin Province), represent long-day photoperiod locations. From lower latitude to higher latitude, the geographical order of seven geographical locations is NB, ZZ, CZ, TY, SY, CY, and GZL (Figure 1A). The trials varied in photoperiod, temperature, and other environmental variables, while the field managements were the same among different trials.

\section{Phenotype evaluation}

When panicles and grains are fully matured, panicle length (PL) was measured with the main panicle, panicle diameter (PD) was measured at the thickest location of the main panicle. Panicle exsertion length (PEL) was measured from the uppermost node to panicle base. Harvested panicles were air-dried and stored at room temperature for 1 month, then primary branches were removed from the panicle, and grains were removed from the branches for measurements. Primary branch length (PBL) was measured by a ruler. Panicle weight (PW), grain weight per panicle (GWP), and 1000-grain weight (TGW) were evaluated using a Mettler-Toledo analytical balance. The number of primary branches (PBN) of one panicle and grain number per primary branch (GNB) were counted by manual. PL and PEL were measured in all of the 13 environments. PD, PW, and GWP were evaluated in 12, 11, and 10 environments, respectively. PBN and TGW were measured in 2 environments, $\mathrm{PBL}$ and GNB were measured in one environment (Figure 1). All traits were measured with three to five replicates.

\section{Statistical analysis of panicle phenotypic variations}


All of the phenotypic variations of the RIL population, including the mean value, standard deviation, skewness, kurtosis, as well as the broad sense heritability $\left(h^{2}\right)$ and correlation analysis, were performed using R packages. Analysis of variance (ANOVA) was carried out to test the statistical significance of various environments of RIL variation for each trait. Variance components corresponding to broad sense heritability were estimated using a mixed linear model using the R package Ime4. Broad sense heritability was calculated by the following formula:

$$
h^{2}=\frac{V_{G}}{\left(V_{G}+\frac{V_{G \times L}}{n_{L}}+\frac{V_{G \times Y}}{n_{Y}}+\frac{V_{r}}{n_{L} \times n_{Y}}\right)}
$$

Where $V_{G}$ is the genotypic variance, $V_{G \times L}$ is the genotype and location interaction variance, $V_{G \times Y}$ is the genotype and year interaction variance, $V_{r}$ is residual variance, $n_{L}$ and $n_{Y}$ are the number of location and year, respectively.

\section{QTL mapping and candidate gene identification}

The high-density genetic map constructed by He et al. (2020) was used in this study. R/qtl package was used to perform QTL mapping by using CIM model with scanning window size of $5 \mathrm{cM}$. The loci with LOD (logarithm of odds ratio) over 2.5 was considered as a QTL and the confidence intervals were estimated using 1.5 LOD-drop method (He et al. 2020). QTL across different environments for the same trait were considered to be the same when the supporting intervals were overlap and the additive effects were originated from the same parental line. The overlapping genomic regions for QTL controlling different traits were considered to be co-located QTL clusters. QTL nomenclature followed the rule described by Mccouch et al. (1997), and alphabetic order was used for QTL on the same chromosome. QTL with a positive or negative additive effect for a specific trait indicate that the increase in the phenotypic value of the trait is contributed by the alleles from Liaogu1 or Ai88, respectively. The genes located in QTL intervals homologous to rice or maize panicle architecture related genes were predicated as the candidate gene.

\section{Results}

\section{Phenotypic variation and broad sense heritability}

In this study, all of the nine traits exhibited diverse phenotypic variations and obvious transgressive segregations in the RIL population (Figure S1 and Table S1). All phenotypes in the RIL population showed normal curve distributions, suggesting that these panicle architecture related traits were under control of quantitative trait loci. The performances of PW, GWP, PL, and PD were influenced by geographical locations (Figure 1B-E and Table S1). The phenotypic variations of the RIL population were quite stable across years at a given location, except for PD and PEL grown in 2017_CY and 2018_CY. Moreover, the average phenotypic values of PL, PD, PW, and GWP in the RIL population increased dramatically from the lower latitude locations to the higher latitude locations. For example, the RIL population grown in 2018_GZL (26.31 cm) had the largest mean value of PL, followed by 2016_GZL $(24.91 \mathrm{~cm}), 2016 \_C Z(23.61 \mathrm{~cm}), 2017 \_C Y(22.90 \mathrm{~cm}), 2018 \_C Y(22.44 \mathrm{~cm})$, 2017_CZ (21.91 cm), 2017_TY (21.01 cm), 2018_SY $(20.17$ cm), 2018_ZZ (18.05 cm), 2017_ZZ (17.44 cm), 2015_NB (17.31 cm), 2016_NB (16.26 cm), and 2017_NB (14.19 cm) (Figure 1D and Table S1). Similar trends were observed for PW, GWP and PD (Figure 1B, C, and E). However, no significant correlation was observed between the latitude and panicle exsertion length (Figure 1F).

Since most panicle architecture related traits tested in this study displayed significant variations in different environments, we evaluated the broad sense heritability $\left(h^{2}\right)$ of PW, GWP, PL, PD, and PEL, which measured the RIL phenotypic data in 6 to 7 locations across 3 to 4 years (Table 1). PL exhibited the highest heritability $\left(h^{2}=0.93\right)$, while GWP showed the lowest heritability $\left(h^{2}=0.34\right)$. The heritability of PW, PD and PEL was $0.40,0.90$, and 0.85 , respectively.

\section{Correlation analysis}

We investigated the relationship between these panicle architecture related traits in each of the 13 environments (Figure S1). PL, PD, and PEL were evaluated in all of the 13 environments except for PD that was not measured in 2016_CZ. PL was positively correlated with PD and PEL in 7 and 9 environments $(p<0.05)$, respectively. PD and PL was positively correlated with PW and GWP in all environments tested ( $p<0.01)$. PW and GWP were evaluated in 11 and 10 environments, respectively. Obviously, PW was highly positively correlated with GWP in all environments tested $(p<0.001)$. We analyzed the correlation of PEL and PW, PEL and GWP in 11 and 10 environments, respectively, finding that PEL was positively correlated with PW and GWP in 2016_NB, 2017_ZZ, 2018_ZZ, and 2016_GZL except that PEL was negatively correlated with PW in 2017_CY $(p<0.05)$. We measured the TGW and GNB in 2017_TY. TGW was positively correlated with PL, PD, PW, and GWP $(p<0.05)$. GNB was positively correlated with PD, PW, and GWP $(p<0.05)$. PBN was positively correlated with PL in 2017_TY and 2018_CY $(p<0.01)$, and positively correlated with PW and GWP in 2017_TY $(p<0.001)$. PBL was positively correlated with PL, PD, PW, and GWP in 2018_CY $(p<0.05)$, while showed negative correlation with PEL in 2018_CY $(p<0.05)$. No significant correlations were observed for other panicle architecture related 
traits in this study. All in all, PL, PD, TGW, GNB, PBN, and PBL were positively correlated with PW and GWP; PEL was negatively correlated with $\mathrm{PD}$ in most environments investigated.

\section{QTL mapping}

One hundred and fifty-nine QTL derived from 239 loci for nine traits were detected under 13 environments across four years (Table S2). The LOD value of these QTL ranged from 2.51 to 22.36 , explained $0.29-25.55 \%$ of the phenotypic variations. Thirty-four out of the 159 QTL were identified in 2 to 12 environments (Table S2). We also compared the genomic intervals of each QTL controlling different traits, there were 17, 14, 1 and 2 genomic intervals shared by 2, 3, 4, and 5 QTL for different traits, respectively (Table S3 and Figure S2).

\section{Panicle weight}

Twenty-four QTL associated with panicle weight were detected in 11 environments across 3 years, explaining $0.58 \%$ to $8.69 \%$ of the phenotypic variations (Table S2). Of these, $q P W 2.6, q P W 3.1, q P W 4.2, q P W 6.2$, and $q P W 7.2$ were detected in two environments, the additive effect of $q P W 3.1$ and $q P W 7.2$ were come from Liaogu1, while favorable alleles of $q P W 2.6, q P W 4.2$, and $q P W 6.2$ were originated from Ai88. The other 19 QTL for PW were only identified under one environment, the additive effect of 13 QTL were from Liaogu1, while others came from Ai88.

\section{Grain weight per panicle}

Sixteen QTL were mapped for grain weight per panicle across nine environments, explained 2.70\%-7.58\% of the phenotypic variations. Of them, qGWP4. 1 was identified in two environments, the favorable alleles were originated from Ai88. The remaining fifteen QTL were only identified in a single environment, the additive effect of the seven QTL were derived from Liaogu1.

\section{Panicle length}

Thirty-five QTL associated with panicle length were mapped on all chromosomes under 13 environments, accounting for $0.29-25.55 \%$ of the phenotypic variations. Of them, $q P L 7.2$ and $q P L 9.5$ were identified across 12 and 7 environments, respectively, additive effect of the two QTL were contributed by Liaogu1. $q P L 9.5$ accounted for $14.95-25.55 \%$ of phenotypic variations. $q P L 5.2$ was identified in three environments, and the favorable allele was originated from Liaogu1. $q P L 2.6, q P L 3.1, q P L 3.8, q P L 4.3, q P L 5.1, q P L 7.3, q P L 9.2$, and $q P L 9.3$ were detected in two environments, all additive effect for panicle length were derived from Liaogu1 except $q P L 3.8$ and $q P L 5.1$ were from Ai88. Remaining twentyfour QTL of panicle length were identified in a single environment, additive effect of twelve QTL were from Liaogu1 and others originated in Ai88.

\section{Panicle diameter}

Twenty-seven QTL for panicle diameter located on all chromosomes except chromosomes 4 and 7 were detected under 12 environments across 4 years, accounting for $0.72-16.34 \%$ of the phenotypic variations. Among them, $q P D 3.1, q P D 6.4$, and $q P D 9.2$ were identified across 5,3 and 10 environments, respectively. The additive effect of $q P D 3.1$ and $q P D 9.2$ were contributed from Liaogu1 and the favorable allele of qPD6.4 was from Ai88. $q P D 9.2$ explained 4.33-16.34\% of the phenotypic variations across 10 environments. $q P D 2.4, q P D 3.4$, and $q P D 5.3$ were detected in two environments, the remaining 21 QTL were identified in single environment.

\section{Panicle exsertion length}

Thirty-five QTL associated with panicle exsertion length mapped on all chromosomes across 13 environments, explained $1.84-11.59 \%$ of the phenotypic variances. Among them, qPEL 1.5, qPEL 1.7, qPEL 5.5, qPEL9.3, and qPEL9.5 were identified under 6, 3, 5, 6, and 8 environments, all additive effect for increasing the PEL were originated from Liaogu1. qPEL3.1, qPEL5.7, qPEL6.1, qPEL6.2, and qPEL9.1 were detected under two environments. Additive effect of $q P E L 3.1$ and $q P E L 9.1$ were derived from Liaogu1, while qPEL5.7, qPEL6.1, and qPEL6.2 were contributed by Ai88. The remaining 25 QTL were identified in only a single environment.

\section{Panicle primary branch length, primary branch number, grain number per branch, and 1000-grain weight}

Four QTL for PBL were mapped on chromosomes 2, 3, and 4 in 2018_CY, explaining 2.88-5.49\% of the phenotypic variations. The additive effect of $q P B L 3.1$ and $q P B L 3.2$ were originated from Liaogu1, and $q P B L 2$ and $q P B L 4$ were from Ai88.

Seven QTL for PBN were identified in 2017_TY, accounting for 0.44-8.86\% of the phenotypic variances. Of them, favorable alleles for increasing the PBN of $q P B N 2.2, q P B N 5$, $q P B N 9.1, q P B N 9.2$, and $q P B N 9.3$ were derived from Liaogu1, while $q P B N 2.1$ and $q P B N 6$ came from Ai88. 
Only one QTL for GNB was detected in 2017_TY, accounting for 3.47\% of the phenotypic variations, the additive effect of qGNB9 was from Liaogu1.

Ten QTL for TGW located on chromosomes 1, 2, 4, 7, 8, and 9 were identified in 2017_TY and 2018_GZL, explained 2.97-10.55\% of the phenotypic variances. Of these, favorable alleles for increasing the TGW of qTGW1.1, qTGW1.2, qTGW2.1, qTGW2.2, qTGW4.1, qTGW7, qTGW8, and $q T G W 9$ were contributed by Liaogu1, while favorable alleles of $q T G W 2.3$ and $q T G W 4.2$ were from Ai88. qTGW4.2 was detected in 2017_TY and 2018_GZL, explained 3.20\% and 3.49\% of the total phenotypic variance, respectively.

\section{Stable QTL and co-locations of QTL for multiple traits}

Thirty-four out of 159 QTL were detected in at least two environments (Figure 2 and Table S2). Twenty- three QTL for PW, GWP, PL, PD, PEL, and TGW were identified in two environments. Three QTL, including $q P L 5.2, q P D 6.4$, and $q P E L 1.7$ were identified in three environments. qPD3. 1 and $q P E L 5.5$ were identified in 5 environments. qPEL 1.5 and $q P E L 9.3$ were detected in 6 environments. qPL9.5, qPEL9.5, qPD9.2, $q P L 7.2$ were detected in $7,8,10$, and 12 environments, respectively.

Phenotypically correlated traits often map to similar genome regions (Zhang et al. 2017). Here, 88 out of 159 QTL controlling different traits formed 34 co-located QTL clusters (Figure S2 and Table S3). Of these, 17 were double co-located QTL, including $q P D 2.1 / q P L 2.3$, qPW2.2/qPL2.5, qTGW2.1/qPBN2.2, qPD2.2/qTGW2.2, qPL3.2/qPEL3.2, qPL3.4/qPD3.7, qPW3.3/qGWP3.3, qPEL4.2/ qPL4.3, qGWP4.2/ qTGW4.2, qPEL5.2/qPBN5, qPL6.2/qPBN6, qPL7.2/qPEL7.2, qPW7.2/qGWP7.1, qTGW7/qGWP7.2, qPL8/qPEL8, qPEL9.1/qPBN9.1, and $q G W P 9.1 / q P B N 9.2$. There were 14 triple co-located QTL, including $q P W 1.2 / q T G W 1.2 / q P E L 1.7, q P L 2.2 / q P B N 2.1 / q P D 2.1$, qPW2.5/qGWP2.2/qPD2.3, qPW2.6/qGWP2.3/qTGW2.3, qPL3.1/qPEL3.1/qPD3.1, qPL3.3/qPD3.5/qPEL3.3, qPW4.2/qGWP4.1/qPL4.3, qPW5/qPL5.3/qPD5.2, qPW6.1/qGWP6.1/qPEL6.1, qPW8/qGWP8/qTGW8, qPW9.1/qPL9.2/qPEL 9.2, qPD9.2/qPL9.6/qGNB9, qPD9.2/qPL9.5/qPEL9.3, and $q P W 9.2 / q G W P 9.2 / q P D 9.3 . q P W 3.1 / q G W P 3.1 / q P D 3.2 / q P B L 3.2$ was a quadruple co-located QTL cluster, qPW6.2/PL6.1/qPD6.3/qGWP6.2/qPD6.4 and qPD9.1/qPL9.3/qTGW9/qPL9.4/qPBN9.3 were quintuple co-located QTL clusters. Intriguingly, the genomic region (chr9: 35,664,032-40,386,141) of $q P D 9.2 / q P L 9.5 / q P E L 9.3$ was detected by 23 times in 13 environments. This triple colocated QTL was a pleiotropic QTL for PD, PL and PEL with large effects, explained 4.33\% ( $q P D 9.2,2016 \mathrm{GZL})$ to $25.55 \%$ ( $q P L 9.5,2016 \mathrm{NB})$ of the phenotypic variations. Moreover, it was close to a triple co-located QTL qPD9.2/qPL9.6/qGNB9 (Chr9: 39,785,988-41,536,123). The close associations between two genomic regions on chromosome 9 with multiple traits suggested the existence of pleiotropy or tight linkage.

\section{Candidate genes}

For candidate gene analysis, we found twenty-five candidate genes located in 22 QTL or co-located QTL clusters are orthologous to panicle development related genes in rice (Table 2). Three candidate genes (Seita.4G281800, Seita.9G281300, and Seita.9G409600) for qGWP4.2, qPW9.1/qPL9.2/qPEL9.2, and qPW9.3 are orthologous to FLORAL ORGAN NUMBER1 (FON1) which regulates floral meristem size and floral organ number in rice. Five genes (Seita.1G328500, Seita.4G077200, Seita.2G002300, Seita.2G383000, and Seita.9G342700) for qPW2.1, qPW2.6, qTGW2.3, qPL4.2, qPL 1.2, and qPD9.2/qPL9.5/qPEL9.3, encode MADS-box transcription factors, they might regulate the panicle architecture by specifying meristem identity. Seita.6G051500 for $q P D 6.2$ is orthologous to the ABERRANT SPIKELET AND PANICLE 1 (ASP1), which is involved in the regulation of meristem fate (Yoshida et al. 2012). Seita.4G185300 for $q G W P 4.1 / q P L 4.3 / q P W 4.2$ is orthologous to ABERRANT PANICLE ORGANIZATION 1 (APO1), which temporally regulates meristem identity, loss of function mutations in APO1 display a precocious conversion of inflorescent meristems to spikelet meristems and have reduced number of primary branches and spikelets (Ikeda et al. 2005). Seita.9G222400 for qPBN9.2 is orthologous to the TAWAWA1 (TAW1) in rice (Yoshida et al. 2013); TAW1 regulates panicle architecture through the suppression of meristem phase transition. Seita.2G219800, Seita.6G171500, and Seita.9G369300 for qPD2.2/qTGW2.3, qPD6.5, and qPD9.3/qPEL9.4, are orthologous to the DENSE AND ERECT PANICLE 1 (DEP1) gene of rice (Huang et al. 2009a), Seita.2G388700 is similar to the OSDEP2 (Zhu et al. 2010), they might regulate of panicle erectness, panicle length and grain size in foxtail millet. Seita.7G126900 for $q P L 7.2$ and Seita.5G243100 for $q P W 5 / q P L 5.3 / q P D 5.2$ encode proteins that are involved in auxin biosynthesis (Abu-Zaitoon 2014; Sazuka et al. 2009; Zhang and Yuan 2014). Seita.4G101300, Seita.1G317400, Seita.5G241500, and Seita.3G136000 for $q P L 4.2, q P L 1.2$, qPW5/qPL5.3/qPD5.2, and qPW3.1/qGWP3.1/qPD3.2/qPBL3.2, encode putative auxin efflux carrier components, they might regulate PW, PL, PD, and PBL via the auxin signaling pathways (Xu et al. 2005). Seita.5G14030 for $q P B N 5 / q P E L 5.2$ is orthologous to the GRAIN NUMBER 1A/Cytokinin oxidase 2 (Gn1a/ OsCKX2) gene of rice, a major QTL contributing to grain number improvement (Ashikari et al. 2005). Seita.9G064000 for qPEL 9.1 encodes a zinc finger transcription factor DROUGHT AND SALT TOLERANCE (DST), which directly regulates OSCKX2 expression in the reproductive meristem (Huang et al. 2009b; Li et al. 2013). Seita. 9 G004400 for qPBN9.1 is orthologous to the Grain Number per Panicle1 (GNP1) that encodes a gibberellin 20 oxidase 1 (GA20ox1) involved in GA degradation pathway (Wu et al. 2016).

\section{Discussion}


Foxtail millet has many excellent characteristics as a model system for $\mathrm{C}_{4}$ plants, because of its small diploid genome, short growth duration, self-fertility, fertile seed setting with small morphological stature, and easy lab management (Doust et al. 2009). In the present study, we investigated the foxtail millet phenotypic variations and the broad sense heritability of panicle architecture and grain yield traits of a RIL population grown in 13 environments. We found that PW, GWP, PL, and PD increased with the increasing latitude (Figure 1). This was in accordance with the increasing day time from south China to north China, suggesting that longer day time contributes to foxtail millet panicle development and enhances grain yield. Zhang et al. (2017) observed similar phenomenon that the mean values of PW, PL, PBN, and GNB at the long-day photoperiod conditions (Zhangjiakou, Hebei province) were greatly reduced at short days (Sanya, Hainan province). Since the fact that panicle development is highly affected by environments differential in photoperiod and other factors, we evaluated the broad sense inheritability of PL, PD, PW, GWP, and PEL, finding that the heritability of PL $\left(h^{2}=0.927\right)$ and PD $\left(h^{2}=0.893\right)$ were higher than that of PW

$\left(h^{2}=0.403\right)$ and GWP $\left(h^{2}=0.337\right)$ (Table 1). This result was in accordance with the studies that the inheritance of PL and PD had higher inheritability of $>70 \%$ than that of TGW, PW, and GWP (39.8\%-59\%) regardless of different environments (Zhao and Lin 1985) (Diao and Jia 2017). Additionally, correlations of nine panicle architecture and yield related traits were analyzed in 13 environments. PL, PD, TGW, GNB, PBN, and PBL were positively correlated with PW and GWP in most environments investigated, this result indicates that improvement of TGW, GNB, PNB, and PBL, especially PL and PD with high heritability $\left(h^{2}>89 \%\right)$, is an alternative way to increase foxtail millet grain yield.

\section{Comparison the QTL identified in this study with previous studies}

We compared the genomic regions of the QTL identified in this study with the QTL detected in other bi-parental populations. Twelve QTL were close to previous studies (Table S2). qPW2.6, qPD8.2, and $q P E L 5.6$ were located close to $q P W 2, q P D 8-2$, and $q N L 5$ identified in a $\mathrm{F}_{2}$ population in foxtail millet including 543 lines from a cross between Aininghuang and Jingu 21 (Wang et al. 2019). Eight QTL, including $q P W 6.1$, qPL4.2, qPL4.3, qPL5.2, qPD2.2, qPD5.3, qPBN2.1, and qPEL9.5, were close to the genomic regions of qpw6, qp/4-1, qp/4-2, qp/5-1, qpd2, qpd5, qcn2-1, and $q n / 9$ that were isolated from a foxtail millet population of 439 RILs (Zhang et al. 2017). qGWP3.3 for grain weight per panicle identified in the present study was located close to the position of qGWP3.3 that was detected in a RIL population from a cross between Longgu7 and Yugu1 (Liu et al. 2020). qPD5.3 for panicle diameter was also close to qMPD5.2 for main panicle diameter detected in a Yugu $1 \times$ Longgu7 $\mathrm{F}_{2}$ population (Fang et al. 2016). Six QTL were nearby to the QTL genomic region identified in our previous study with a natural population of 916 accessions (Jia et al. 2013). qPL 1.2, qPL2.6, and qPL7.1 for panicle length were close to QTL (Chr1: 37,343,439 and 37,378,964), (chr2: 22,845,341), and (chr7: 21,691,982), respectively. The genomic interval of $q P L 9.5$ covered the genomic regions of two QTL for panicle length (Chr9: 38,568,427 and 39,540,370). qPD6.3 and qPD6.4 were close to the QTL (chr6: 8,035,171) and (chr6: 22,571,518) for panicle diameter (Jia et al. 2013).

\section{Candidate gene analysis for panicle architecture or grain yield related QTL}

In grass species, the basic architecture of inflorescence (panicle) is defined by the spatial arrangement of spikelets, which are small branches containing a variable number of flowers. During panicle development, the inflorescence meristem (IM) produces a number of primary branch meristems (PBMs), PBMs differentiate into secondary branch meristems (SBMs), some SBMs may form several tertiary branches, each of the tertiary branches bears a certain number of spikelet meristems (SMs), finally SMs form grains (Xing and Zhang 2010; Yoshida et al. 2013). Many genes involved in specifying inflorescence meristem identity have been cloned. ASP1 encodes a TOPLESS-related transcriptional corepressor that is involved in the regulation of meristem fate in rice. A recessive aps 1 mutant displays a disorganized branching pattern and aberrant spikelet morphology (Yoshida et al. 2012). APO1 temporally regulates meristem identity in rice, the IM of apo1 was converted to a SM after producing a small number of PBMs, resulting in small panicles (Ikeda et al. 2005). OsMADS14, OsMADS15 and OsMADS18 are APETALA1 (AP1)/FRUITFULL (FUL)-like genes that involve in inducing the transition from shoot apical meristem to inflorescence meristem in rice, triple knockdown plants in pap2 mutant inhibited the transition of the meristem to the IM (Kobayashi et al. 2012). We identified 4 candidate genes that are orthologous to $A S P 1, A P 01$, OsMADS15, and OsMADS18 of rice, in the genomic regions of $q P D 6.2$, qGWP4.1/qPL4.3/qPW4.2, qPW2.1, and $q P W 2.6 / q T G W 2.3$, respectively, suggesting that these genes might play essential roles in regulating PW, GWP, TGW, and PL in foxtail millet. Moreover, delays in SM specification lead to iterations of branching, resulting in larger panicles that could potentially produce more grain. In the dominant gain-of-function mutant tawawa 1-D, the activity of the IM is extended and spikelet specification is delayed, resulting in prolonged branch formation and increased numbers of spikelets (Yoshida et al. 2013). We identified a gene (Seita.9G222400) that is orthologous to the rice TAWAWA1 in the qPBN9.2 for primary branch number, suggesting that this gene may play a role in the formation of primary branch number in foxtail millet. OsMADS22 and OsMADS56 regulate inflorescence branching by repressing the expression RCN4 in rice (Liu et al. 2013). SiMADS56 (Seita.9G342700) is located in the genomic region of qPL9.6/qPD9.2/qPEL9.3, which was detected by 23 times in 13 environments for three traits, suggesting that SiMADS56 may play an essencial role in regulating branching in foxtail millet that independent of environments.

\section{Phytohormone plays important role in foxtail millet panicle architecture and grain yield}


Auxin plays a key role in determining axillary meristem initiation and outgrowth. Seita.5G243100 and Seita.7G126900 are orthologous to auxin biosynthesis genes of YUC1 and TDD1 in rice. Seita.1G317400, Seita.3G136000, Seita.4G101300, and Seita.5G241500 encode putative auxin efflux carrier proteins that involved in auxin transport. Cytokinin and GAs play antagonistic roles in regulating reproductive meristem activity, increased cytokinin activity leads to higher grain number, whereas GAs negatively affect meristem activity. In the genomic regions of qPBN5/ qPEL5.2, we identified a gene (Seita.5G140300) is orthologous to Gn1a, which encodes cytokinin oxidase/dehydrogenase (OsCKX2) that degrades cytokinin (Ashikari et al. 2005). Intriguingly, we also detected a rice DST ortholog (Seita.9G064000) in qPEL9.1, DST enhances grain production through controlling Gn1a/OsCKX2 expression. Seita.9G004400, located in the genomic region of qPBN9.1, was orthologous to the rice GNP1 which encodes a GA20ox1 protein. KNOX proteins function as modulators, balance cytokinin and GA activity in the meristem, increased expression of the GA catabolism genes GA2oxs in NIL-GNP1 ${ }^{\mathrm{TQ}}$ decreased $\mathrm{GA}_{1}$ and $\mathrm{GA}_{3}$ accumulation, resulting in increased cytokinin activity, consequently improved grain number and yield (Wu et al. 2016).

\section{Declarations}

\section{Founding}

This work was supported by National Key R\&D Program of China (grant nos. 2019YFD1000700, and 2019YFD1000701), China Agricultural Research System (CARS06-13.5), National Natural Science foundation of China (31871630), China Postdoctoral Science Foundation (2018M641553), and the Agricultural Science and Technology Innovation Program of the Chinese Academy of Agricultural Sciences.

\section{Conflict of interest}

The authors declare no conflict of interest.

\section{Authors' contributions}

Hui Zhi and Qiang He and did data analysis and drafted the manuscript. Xianmin diao and Hui Zhi designed the experiment, developed the RIL population, and revised the manuscript. Jun Liu helped in the data analysis and discussion. Sha Tang, Junjun Yang, Wei Zhang, Huifang Liu, Yanchao Jia, Guanqing Jia, Aiying Zhang, Yuhui Li, Erhu Guo, Ming Gao, Shujie Li, Junxia Li, Na Qin, Cancan Zhu, Chunye Ma, Haijin Zhang, Guoqiu Chen, Wenfei Zhang, Haigang Wang, Zhijun Qiao, Lu Xing, Suying Wang, and Jinrong Liu collected the phenotype. All authors have read and approved the final manuscript.

\section{Acknowledgement}

This work was supported by National Key R\&D Program of China (grant nos. 2019YFD1000700, and 2019YFD1000702), China Agricultural Research System (CARS06-13.5), National Natural Science foundation of China (31871630), China Postdoctoral Science Foundation (2018M641553), and the Agricultural Science and Technology Innovation Program of the Chinese Academy of Agricultural Sciences.

\section{References}

1. Abu-Zaitoon YM (2014) Phylogenetic analysis of putative genes involved in the tryptophan-dependent pathway of auxin biosynthesis in rice. Appl Biochem Biotechnol 172:2480-2495

2. Andrea, Gallavotti, Qiong, Zhao, Junko, Kyozuka, Robert, B., Meeley, Matthew (2004) The role of barren stalk1 in the architecture of maize. Nature 432

3. Ashikari M, Sakakibara H, Lin S, Yamamoto T, Takashi T, Nishimura A, Angeles ER, Qian Q, Kitano H, Matsuoka M (2005) Cytokinin oxidase regulates rice grain production. Science 309:741-745

4. Bommert P, Lunde C, Nardmann J, Vollbrecht E, Running M, Jackson D, Hake S, Werr W (2005) Thick tassel dwarf1 encodes a putative maize ortholog of the Arabidopsis CLAVATA1 leucine-rich repeat receptor-like kinase. Development (Cambridge, England) 132:1235-1245

5. Chu H, Qian Q, Liang W, Yin C, Tan H, Yao X, Yuan Z, Yang J, Huang H, Luo D, Ma H, Zhang D (2006) The Floral Organ Number4 gene encoding a putative ortholog of Arabidopsis CLAVATA3 regulates apical meristem size in rice. Plant physiology 142:1039-1052

6. Danilevskaya ON, Meng X, Ananiev EV (2010) Concerted modification of flowering time and inflorescence architecture by ectopic expression of TFL1-like genes in maize. Plant Physiol 153:238-251

7. Diao X, Jia G (2017) Foxtail Millet Germplasm and Inheritance of Morphological Characteristics. Springer International Publishing

8. Diao X, Schnable J, Bennetzen JL, Jiayang L (2014) Initiation of Setaria as a model plant. Frontiers of Agricultural Science and Engineering 1:16-20 
9. Doust A (2007) Architectural evolution and its implications for domestication in grasses. Ann Bot 100:941-950

10. Doust AN, Devos KM, Gadberry MD, Gale MD, Kellogg EA (2005) The genetic basis for inflorescence variation between foxtail and green millet (poaceae). Genetics 169:1659-1672

11. Doust AN, Kellogg EA, Devos KM, Bennetzen JL (2009) Foxtail millet: a sequence-driven grass model system. Plant Physiol 149:137-141

12. Fang X, Dong K, Wang X, Liu T, He J, Ren R, Zhang L, Liu R, Liu X, Li M, Huang M, Zhang Z, Yang T (2016) A high density genetic map and QTL for agronomic and yield traits in Foxtail millet [Setaria italica (L.) P. Beauv]. BMC Genomics 17:336

13. Gao X, Liang W, Yin C, Ji S, Wang H, Su X, Guo C, Kong H, Xue H-W, Zhang D (2010) The SEPALLATA-like gene OsMADS34 is required for rice inflorescence and spikelet development. Plant physiology 153:728-740

14. He Q, Zhi H, Tang S, Xing L, Wang S, Wang H, Zhang A, Li Y, Gao M, Zhang H, Chen G, Dai S, Li J, Yang J, Liu H, Zhang W, Jia Y, Li S, Liu J, Qiao Z, Guo E, Jia G, Liu J, Diao X (2020) QTL mapping for foxtail millet plant height in multi-environment using an ultra-high density bin map. Theor Appl Genet

15. Hu H, Mauro-Herrera M, Doust AN (2018) Domestication and Improvement in the Model C4 Grass, Setaria. Front Plant Sci 9:719

16. Huang P, Jiang H, Zhu C, Barry K, Jenkins J, Sandor L, Schmutz J, Box MS, Kellogg EA, Brutnell TP (2017) Sparse panicle1 is required for inflorescence development in Setaria viridis and maize. Nat Plants 3:17054

17. Huang X, Qian Q, Liu Z, Sun H, He S, Luo D, Xia G, Chu C, Li J, Fu X (2009a) Natural variation at the DEP1 locus enhances grain yield in rice. Nat Genet 41:494-497

18. Huang XY, Chao DY, Gao JP, Zhu MZ, Shi M, Lin HX (2009b) A previously unknown zinc finger protein, DST, regulates drought and salt tolerance in rice via stomatal aperture control. Genes Dev 23:1805-1817

19. Hussin SH, Wang H, Tang S, Zhi H, Tang C, Zhang W, Jia G, Diao X (2020) SiMADS34, an E-class MADS-box transcription factor, regulates inflorescence architecture and grain yield in Setaria italica. Plant Mol Biol

20. Ikeda K, Nagasawa N, Nagato Y (2005) ABERRANT PANICLE ORGANIZATION 1 temporally regulates meristem identity in rice. Dev Biol 282:349-360

21. Jaiswal V, Gupta S, Gahlaut V, Muthamilarasan M, Bandyopadhyay T, Ramchiary N, Prasad M (2019) Genome-Wide Association Study of Major Agronomic Traits in Foxtail Millet (Setaria italica L.) Using ddRAD Sequencing. Sci Rep 9:5020

22. Jia G, Huang X, Zhi H, Zhao Y, Zhao Q, Li W, Chai Y, Yang L, Liu K, Lu H, Zhu C, Lu Y, Zhou C, Fan D, Weng Q, Guo Y, Huang T, Zhang L, Lu T, Feng Q, Hao H, Liu H, Lu P, Zhang N, Li Y, Guo E, Wang S, Wang S, Liu J, Zhang W, Chen G, Zhang B, Li W, Wang Y, Li H, Zhao B, Li J, Diao $X$, Han B (2013) A haplotype map of genomic variations and genome-wide association studies of agronomic traits in foxtail millet (Setaria italica). Nat Genet 45:957-961

23. Kobayashi K, Yasuno N, Sato Y, Yoda M, Yamazaki R, Kimizu M, Yoshida H, Nagamura Y, Kyozuka J (2012) Inflorescence meristem identity in rice is specified by overlapping functions of three AP1/FUL-like MADS box genes and PAP2, a SEPALLATA MADS box gene. The Plant cell 24:1848-1859

24. Komatsu K, Maekawa M, Ujiie S, Satake Y, Furutani I, Okamoto H, Shimamoto K, Kyozuka J (2003) LAX and SPA: major regulators of shoot branching in rice. Proc Natl Acad Sci U S A 100:11765-11770

25. Komatsu M, Maekawa M, Shimamoto K, Kyozuka J (2001) The LAX1 and FRIZZY PANICLE 2 genes determine the inflorescence architecture of rice by controlling rachis-branch and spikelet development. Dev Biol 231:364-373

26. Kurakawa T, Ueda N, Maekawa M, Kobayashi K, Kojima M, Nagato Y, Sakakibara H, Kyozuka J (2007) Direct control of shoot meristem activity by a cytokinin-activating enzyme. Nature 445:652-655

27. Kyozuka J, Tokunaga H, Yoshida A (2014) Control of grass inflorescence form by the fine-tuning of meristem phase change. Curr Opin Plant Biol 17:110-115

28. Li S, Zhao B, Yuan D, Duan M, Qian Q, Tang L, Wang B, Liu X, Zhang J, Wang J, Sun J, Liu Z, Feng YQ, Yuan L, Li C (2013) Rice zinc finger protein DST enhances grain production through controlling Gn1a/OsCKX2 expression. Proc Natl Acad Sci U S A 110:3167-3172

29. Liu C, Teo ZW, Bi Y, Song S, Xi W, Yang X, Yin Z, Yu H (2013) A conserved genetic pathway determines inflorescence architecture in Arabidopsis and rice. Dev Cell 24:612-622

30. Liu T, He J, Dong K, Wang X, Wang W, Yang P, Ren R, Zhang L, Zhang Z, Yang T (2020) QTL mapping of yield component traits on bin map generated from resequencing a RIL population of foxtail millet (Setaria italica). BMC Genomics 21:141

31. Long JA, Moan El, Medford JI, Barton MK (1996) A member of the KNOTTED class of homeodomain proteins encoded by the STM gene of Arabidopsis. Nature 379:66-69

32. Lu H, Zhang J, Liu KB, Wu N, Li Y, Zhou K, Ye M, Zhang T, Zhang H, Yang X, Shen L, Xu D, Li Q (2009) Earliest domestication of common millet (Panicum miliaceum) in East Asia extended to 10,000 years ago. Proc Natl Acad Sci U S A 106:7367-7372 
33. Mccouch S, Cho Y, Yano M, Paul E, Blinstrub M, Morishima H, Mccouch S, Cho Y, Paul E, Morishima H (1997) Report on QTL nomenclature. Rice Genet Newsl 14

34. Nakagawa M, Shimamoto K, Kyozuka J (2002) Overexpression of RCN1 and RCN2, rice TERMINAL FLOWER 1/CENTRORADIALIS homologs, confers delay of phase transition and altered panicle morphology in rice. Plant J 29:743-750

35. Nardmann J, Werr W (2007) The Shoot Stem Cell Niche in Angiosperms: Expression Patterns of WUS Orthologues in Rice and Maize Imply Major Modifications in the Course of Mono- and Dicot Evolution. Molecular biology and evolution 23:2492-2504

36. Phillips KA, Skirpan AL, Liu X, Christensen A, Slewinski TL, Hudson C, Barazesh S, Cohen JD, Malcomber S, McSteen P (2011) vanishing tassel 2 encodes a grass-specific tryptophan aminotransferase required for vegetative and reproductive development in maize. Plant Cell 23:550-566

37. Qiao Y, Piao R, Shi J, Lee SI, Jiang W, Kim BK, Lee J, Han L, Ma W, Koh HJ (2011) Fine mapping and candidate gene analysis of dense and erect panicle 3, DEP3, which confers high grain yield in rice (Oryza sativa L.). Theor Appl Genet 122:1439-1449

38. Sazuka T, Kamiya N, Nishimura T, Ohmae K, Sato Y, Imamura K, Nagato Y, Koshiba T, Nagamura Y, Ashikari M, Kitano H, Matsuoka M (2009) A rice tryptophan deficient dwarf mutant, tdd1, contains a reduced level of indole acetic acid and develops abnormal flowers and organless embryos. Plant J 60:227-241

39. Suzaki T, Sato M, Ashikari M, Miyoshi M, Nagato Y, Hirano H-Y (2004) The gene FLORAL ORGAN NUMBER1 regulates floral meristern size in rice and encodes a leucine-rich repeat receptor kinase orthologous to Arabidopsis CLAVATA1. Development (Cambridge, England) 131:5649-5657

40. Tsuda K, Ito Y, Sato Y, Kurata N (2011) Positive autoregulation of a KNOX gene is essential for shoot apical meristem maintenance in rice. Plant Cell 23:4368-4381

41. Vollbrecht E, Reiser L, Hake S (2000) Shoot meristem size is dependent on inbred background and presence of the maize homeobox gene, knotted1. Development (Cambridge, England) 127:3161

42. Wang J, Wang Z, Du X, Yang H, Han F, Han Y, Yuan F, Zhang L, Peng S, Guo E (2017) A high-density genetic map and QTL analysis of agronomic traits in foxtail millet [Setaria italica (L.) P. Beauv.] using RAD-seq. PLoS One 12:e0179717

43. Wang Z, Wang J, Peng J, Du X, Jiang M, Li Y, Han F, Du G, Yang H, Lian S, Yong J, Cai W, Cui J, Han K, Yuan F, Chang F, Yuan G, Zhang W, Zhang L, Peng S, Zou H, Guo E (2019) QTL mapping for 11 agronomic traits based on a genome-wide Bin-map in a large F2 population of foxtail millet (Setaria italica (L.) P. Beauv). Molecular Breeding 39:18

44. Wu Y, Wang Y, Mi XF, Shan JX, Li XM, Xu JL, Lin HX (2016) The QTL GNP1 Encodes GA20ox1, Which Increases Grain Number and Yield by Increasing Cytokinin Activity in Rice Panicle Meristems. PLoS Genet 12:e1006386

45. Xiang J, Tang S, Zhi H, Jia G, Wang H, Diao X (2017) Loose Panicle1 encoding a novel WRKY transcription factor, regulates panicle development, stem elongation, and seed size in foxtail millet [Setaria italica (L.) P. Beauv.]. PLoS One 12:e0178730

46. Xing Y, Zhang Q (2010) Genetic and molecular bases of rice yield. Annu Rev Plant Biol 61:421-442

47. Xu M, Zhu L, Shou H, Wu P (2005) A PIN1 family gene, OsPIN1, involved in auxin-dependent adventitious root emergence and tillering in rice. Plant Cell Physiol 46:1674-1681

48. Yoshida A, Ohmori Y, Kitano H, Taguchi-Shiobara F, Hirano HY (2012) Aberrant spikelet and panicle1, encoding a TOPLESS-related transcriptional co-repressor, is involved in the regulation of meristem fate in rice. Plant $\mathrm{J}$ 70:327-339

49. Yoshida A, Sasao M, Yasuno N, Takagi K, Daimon Y, Chen R, Yamazaki R, Tokunaga H, Kitaguchi Y, Sato Y, Nagamura Y, Ushijima T, Kumamaru T, lida S, Maekawa M, Kyozuka J (2013) TAWAWA1, a regulator of rice inflorescence architecture, functions through the suppression of meristem phase transition. Proc Natl Acad Sci U S A 110:767-772

50. Zhang D, Yuan Z (2014) Molecular control of grass inflorescence development. Annu Rev Plant Biol 65:553-578

51. Zhang K, Fan G, Zhang X, Zhao F, Wei W, Du G, Feng X, Wang X, Wang F, Song G, Zou H, Zhang X, Li S, Ni X, Zhang G, Zhao Z (2017) Identification of QTLs for 14 Agronomically Important Traits in Setaria italica Based on SNPs Generated from High-Throughput Sequencing. G3 (Bethesda) 7:1587-1594

52. Zhao H, Jin L. Variations and inheritance of quantitative traits in several type of foxtail millet cultivars. In: Academy of Agricultural Sciences in Heilongjiang of China, editor. Alum of foxtail millet studies in Northeast China. Heilongjiang: Academy of Agricultural Sciences; 1985. p. 89-92.

53. Zhu K, Tang D, Yan C, Chi Z, Yu H, Chen J, Liang J, Gu M, Cheng Z (2010) Erect panicle2 encodes a novel protein that regulates panicle erectness in indica rice. Genetics 184:343-350

\section{Tables}

Table 10 Genotype $\times$ Environment interactions and heritability of panicle morphology and grain yield related traits

Page 11/15 


\begin{tabular}{|c|c|c|c|c|c|c|c|c|c|}
\hline \multirow[t]{2}{*}{ Trait } & \multirow{2}{*}{$\begin{array}{l}\text { Location } \\
\text { number }\end{array}$} & \multirow[t]{2}{*}{ Year } & \multicolumn{6}{|l|}{ Variance } & \multirow{2}{*}{$\begin{array}{l}\text { broad sense } \\
\text { heritability }\end{array}$} \\
\hline & & & $\begin{array}{l}\text { Genotype } \times \\
\text { Location }\end{array}$ & $\begin{array}{l}\text { Genotype × } \\
\text { Year }\end{array}$ & Genotype & Location & Year & Residual & \\
\hline PW & 7 & 3 & 0 & 0 & 1.59 & 138.7 & 38.82 & 49.28 & 0.40 \\
\hline GWP & 6 & 3 & 0 & 0 & 1.01 & 79.63 & 18.68 & 35.78 & 0.34 \\
\hline PL & 7 & 4 & 0.32 & 0 & 2.34 & 12.11 & 1.16 & 3.88 & 0.93 \\
\hline PD & 7 & 4 & 0 & 0 & 4.52 & 13.55 & 3.89 & 15.21 & 0.90 \\
\hline PEL & 7 & 4 & 0 & 0 & 2.82 & 36.88 & 12.41 & 13.51 & 0.85 \\
\hline
\end{tabular}

Table 2. Candidate genes that located in the genomic regions of QTL 


\begin{tabular}{|c|c|c|c|c|c|}
\hline \multirow[t]{2}{*}{ QTL } & \multirow[t]{2}{*}{ Candidate gene } & \multicolumn{3}{|l|}{ Rice ortholog } & \multirow[t]{2}{*}{ Reference } \\
\hline & & Gene ID & Name & Annotation & \\
\hline \multirow[t]{2}{*}{$q P L 1.2$} & Seita.1G317400 & LOC_Os02g50960.1 & PIN1B & $\begin{array}{l}\text { Auxin efflux carrier } \\
\text { component }\end{array}$ & $\begin{array}{l}\text { Xu et al. } \\
\text { (2005) }\end{array}$ \\
\hline & Seita.1G328500 & LOC_Os02g52340.1 & OsMADS22 & $\begin{array}{l}\text { OsMADS22 - MADS-box } \\
\text { family gene with MIKCc } \\
\text { type-box }\end{array}$ & $\begin{array}{l}\text { Liu et al. } \\
\text { (2013) }\end{array}$ \\
\hline qPW2.1 & Seita.2G002300 & LOC_Os07g01820.3 & OsMADS15 & $\begin{array}{l}\text { OsMADS15 - MADS-box } \\
\text { family gene with MIKCc } \\
\text { type-box }\end{array}$ & $\begin{array}{l}\text { Kobayashi } \\
\text { et al. } \\
\text { (2012) }\end{array}$ \\
\hline qPD2.2/qTGW2.2 & Seita.2G219800 & LOC_Os09g26999.1 & DEP1 & $\begin{array}{l}\text { Phosphatidylethanolamine- } \\
\text { binding protein (PEBP) like } \\
\text { domain protein }\end{array}$ & $\begin{array}{l}\text { Huang et } \\
\text { al. } \\
(2009 a)\end{array}$ \\
\hline qPW2.6/qTGW2.3 & Seita.2G383000 & LOC_Os07g41370.1 & OsMADS18 & $\begin{array}{l}\text { OsMADS18 - MADS-box } \\
\text { family gene with MIKCc } \\
\text { type-box }\end{array}$ & $\begin{array}{l}\text { Kobayashi } \\
\text { et al. } \\
(2012)\end{array}$ \\
\hline qGWP2.3/qPW2.6/qTGW2.3 & Seita.2G388700 & LOC_Os07g42410.1 & $D E P 2$ & $\begin{array}{l}\text { Hypothetical conserved } \\
\text { gene that regulate of } \\
\text { panicle erectness, panicle } \\
\text { length and grain size }\end{array}$ & $\begin{array}{l}\text { Zhu et al. } \\
\text { (2010) }\end{array}$ \\
\hline qPW3.1/qGWP3.1/qPD3.2/qPBL3.2 & Seita.3G136000 & LOC_Os05g50140.1 & PIN3B & $\begin{array}{l}\text { Similar to PIN1-like auxin } \\
\text { efflux carrier protein }\end{array}$ & $\begin{array}{l}\text { Xu et al. } \\
\text { (2005) }\end{array}$ \\
\hline qGWP3.2/qPD3.3 & Seita.3G189300 & LOC_Os05g42130.1 & & $\begin{array}{l}\text { GRAS transcription factor } \\
\text { domain containing protein } \\
\text { that similar to MONOCULM } \\
1\end{array}$ & \\
\hline \multirow[t]{2}{*}{$q P L 4.2$} & Seita.4G077200 & LOC_Os02g52340.1 & OsMADS22 & $\begin{array}{l}\text { OsMADS22 - MADS-box } \\
\text { family gene with MIKCc } \\
\text { type-box }\end{array}$ & $\begin{array}{l}\text { Liu et al. } \\
(2013)\end{array}$ \\
\hline & Seita.4G101300 & LOC_Os06g12610.1 & PIN1A & $\begin{array}{l}\text { Similar to PIN1-like auxin } \\
\text { efflux carrier protein }\end{array}$ & $\begin{array}{l}\text { Xu et al. } \\
\text { (2005) }\end{array}$ \\
\hline qGWP4.1/qPL4.3 /qPW4.2 & Seita.4G185300 & LOC_Os06g45460.1 & APO1 & $\begin{array}{l}\text { F-box protein, Inflorescence } \\
\text { form, Lodging resistance } \\
\text { and grain yield }\end{array}$ & $\begin{array}{l}\text { Ikeda et } \\
\text { al. (2005) }\end{array}$ \\
\hline qGWP4.2 & Seita.4G281800 & LOC_Os06g50340.1 & FON1 & $\begin{array}{l}\text { Receptor protein kinase } \\
\text { CLAVATA1 precursor }\end{array}$ & $\begin{array}{l}\text { Suzaki et } \\
\text { al. } ₫ 2004)\end{array}$ \\
\hline qPBN5/qPEL5.2 & Seita.5G140300 & LOC_Os01g10110.1 & Gn1a & $\begin{array}{l}\text { Cytokinin } \\
\text { oxidase/dehydrogenase }\end{array}$ & $\begin{array}{l}\text { Ashikari et } \\
\text { al.(2005) }\end{array}$ \\
\hline \multirow[t]{2}{*}{ qPW5/qPL5.3/qPD5.2 } & Seita.5G241500 & LOC_Os01g45550.2 & PIN3A & $\begin{array}{l}\text { Auxin efflux carrier } \\
\text { component }\end{array}$ & $\begin{array}{l}\text { Xu et al. } \\
\text { (2005) }\end{array}$ \\
\hline & Seita.5G243100 & LOC_Os01g45760.1 & YUC1 & $\begin{array}{l}\text { Flavin monooxygenase-like } \\
\text { enzyme }\end{array}$ & $\begin{array}{l}\text { Abu- } \\
\text { Zaitoon } \\
2014\end{array}$ \\
\hline qPD6.2 & Seita.6G051500 & LOC_Os08g06480.1 & $A S P 1$ & $\begin{array}{l}\text { Transcriptional co- } \\
\text { repressor, lissencephaly } \\
\text { type-1-like homology }\end{array}$ & $\begin{array}{l}\text { Yoshida et } \\
\text { al. (2012) }\end{array}$ \\
\hline qPD6.5 & Seita.6G171500 & LOC_Os09g26999.1 & $D E P 1$ & $\begin{array}{l}\text { Phosphatidylethanolamine- } \\
\text { binding protein (PEBP) like } \\
\text { domain protein }\end{array}$ & $\begin{array}{l}\text { Huang et } \\
\text { al. } \\
(2009 a)\end{array}$ \\
\hline$q P L 7.2$ & Seita.7G126900 & LOC_Os04g38950.1 & $T D D 1$ & $\begin{array}{l}\text { Similar to anthranilate } \\
\text { synthase beta chain }\end{array}$ & $\begin{array}{l}\text { Sazuka et } \\
\text { al. (2009) }\end{array}$ \\
\hline qPBN9.1 & Seita.9G004400 & LOC_Os03g63970.1 & GNP1 & Gibberellin 20 oxidase 1 & $\begin{array}{l}\text { Wu et al. } \\
\text { (2016) }\end{array}$ \\
\hline qPEL 9.1 & Seita.9G064000 & LOC_Os03g57240.1 & $D S T$ & $\begin{array}{l}\mathrm{C} 2 \mathrm{H} 2 \text { zinc finger } \\
\text { transcription factor }\end{array}$ & $\begin{array}{l}\text { Li et al. } \\
\text { (2013) }\end{array}$ \\
\hline qPBN9.2 & Seita.9G222400 & LOC_Os10g33780.1 & TAWAWA1 & $\begin{array}{l}\text { DUF640 domain containing } \\
\text { protein }\end{array}$ & $\begin{array}{l}\text { Yoshida et } \\
\text { al. (2013) }\end{array}$ \\
\hline
\end{tabular}

Page 13/15 


\begin{tabular}{|c|c|c|c|c|c|}
\hline qPW9.1/qPL9.2/qPEL 9.2 & Seita.9G281300 & LOC_Os06g50340.1 & FON1 & $\begin{array}{l}\text { Receptor protein kinase } \\
\text { CLAVATA1 precursor }\end{array}$ & $\begin{array}{l}\text { Suzaki et } \\
\text { al. 『2004) }\end{array}$ \\
\hline qPD9.2/qPL9.5/qPEL 9.3 & Seita.9G342700 & LOC_Os10g39130.1 & OsMADS56 & $\begin{array}{l}\text { OsMADS56 - MADS-box } \\
\text { family gene with MIKCc } \\
\text { type-box }\end{array}$ & $\begin{array}{l}\text { Liu et al. } \\
\text { (2013) }\end{array}$ \\
\hline qPD9.3/qPEL9.4 & Seita.9G369300 & LOC_Os09g26999.1 & DEP1 & $\begin{array}{l}\text { Phosphatidylethanolamine- } \\
\text { binding protein (PEBP) like } \\
\text { domain protein }\end{array}$ & $\begin{array}{l}\text { Huang et } \\
\text { al. } \\
\text { (2009a) }\end{array}$ \\
\hline qPW9.3 & Seita.9G409600 & LOC_Os06g50340.1 & FON1 & $\begin{array}{l}\text { Receptor protein kinase } \\
\text { CLAVATA1 precursor }\end{array}$ & $\begin{array}{l}\text { Suzaki et } \\
\text { al. 『2004) }\end{array}$ \\
\hline
\end{tabular}

\section{Figures}

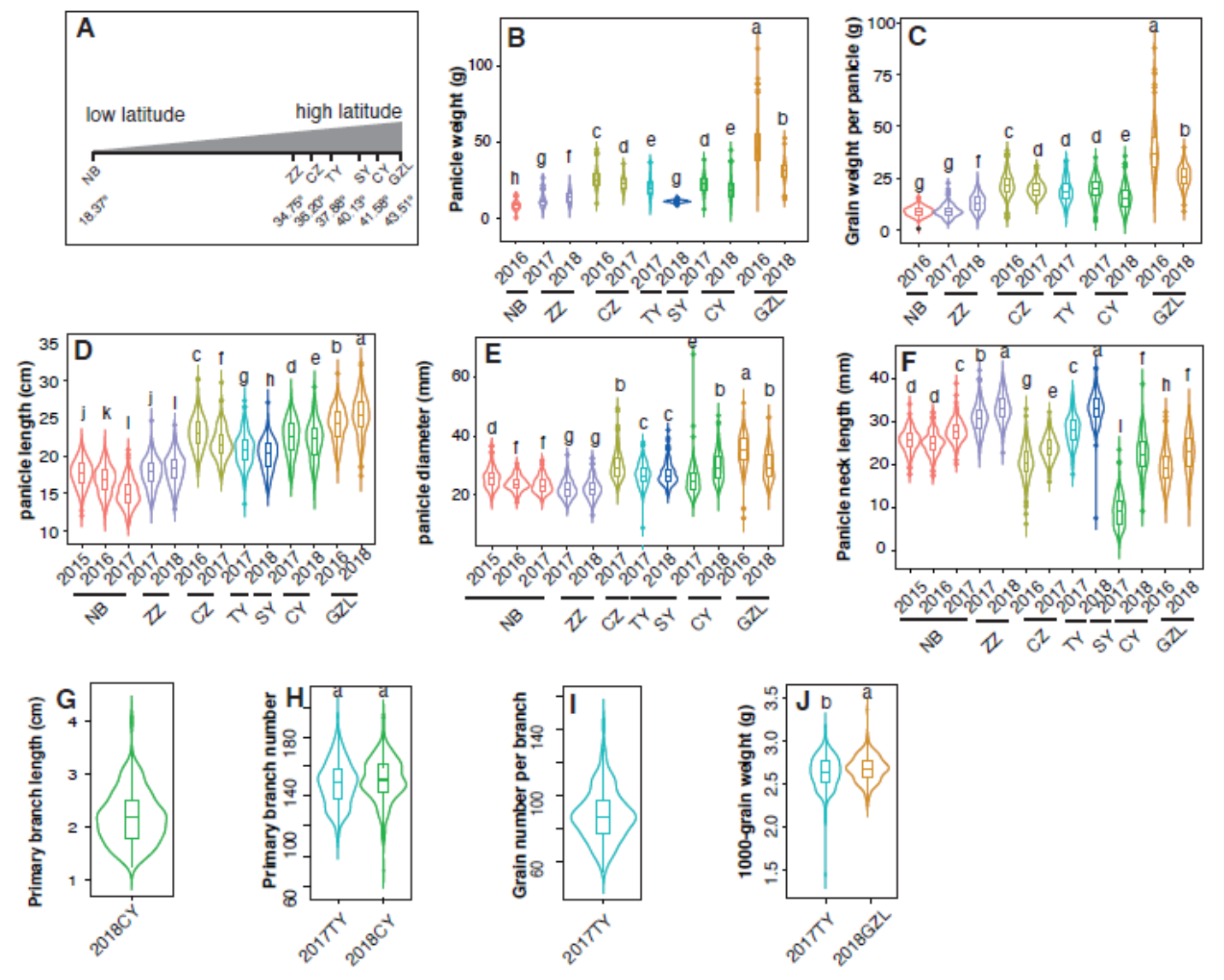

Figure 1

Phenotypic variations of panicle related traits in multi-environments. A) Geographical locations where the RIL population grown. B)Panicle weight, C)Grain weight per panicle, D) Panicle length, E) Panicle diameter, F) Panicle exsertion length, G) Primary branch length, H) Primary branch number, I) Grain number per branch, and J) 1000-grain weight variations of the RILs in different locations. 
Panlcle welght

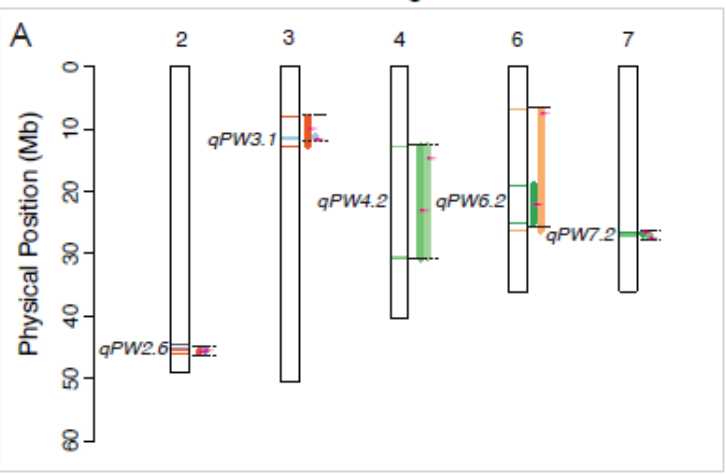

Panlcle dlameter

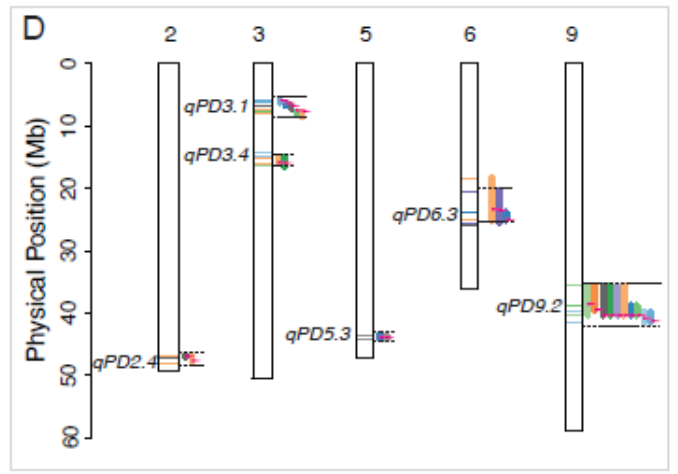

Graln welght per panlcle

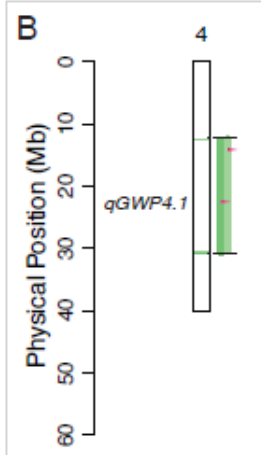

Panlcle length

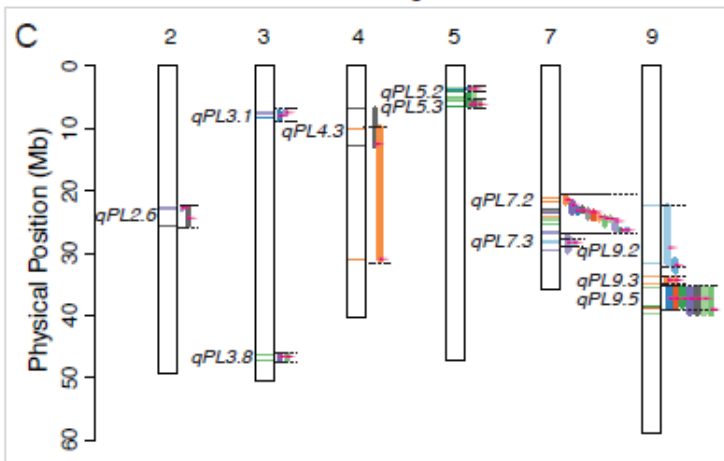

Panlcle exsertion length

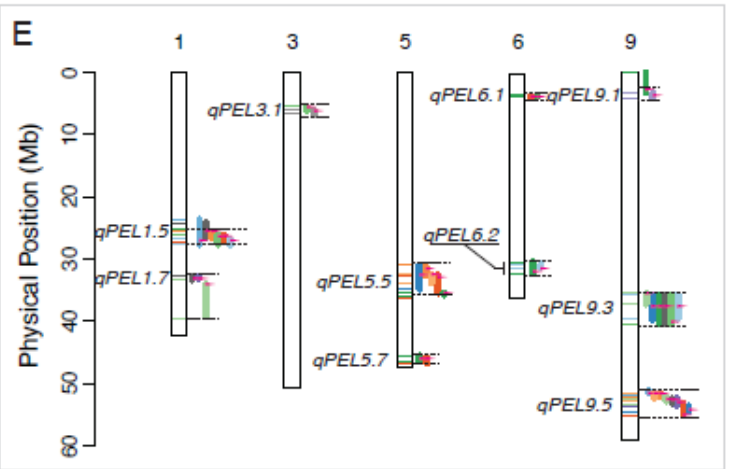

1000-graln welght

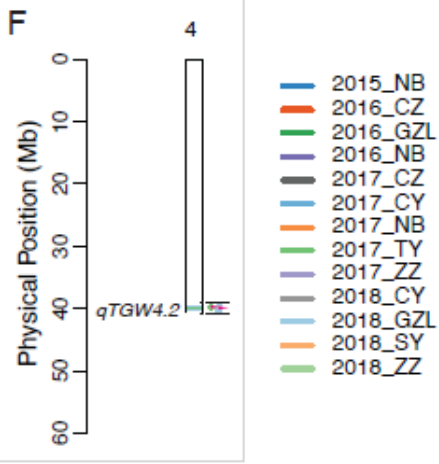

Figure 2

Multi-environment QTL identified for panicle architecture related traits. Dashed black lines delimit common QTL regions defined by overlapping QTL intervals, red arrow points out the peak position of each QTL. A) Panicle weight, B) Grain weight per panicle, C) Panicle length, D) Panicle diameter, E) Panicle exsertion length, F) 1000-grain weight.

\section{Supplementary Files}

This is a list of supplementary files associated with this preprint. Click to download.

- SupplementaryFigures.docx

- Tables1.xlsx

- Tables2.xlsx

- Tables3.xlsx 